\title{
Diabetes Prevention Program (DPP)
}

National Diabetes Information Clearinghouse (NDIC)

\section{Definitions}

Type 2 diabetes

Defined by National Diabetes Information Clearinghouse (NDIC)

Impaired glucose tolerance (IGT)

Defined by National Diabetes Information Clearinghouse (NDIC)

Pre-diabetes

Defined by National Diabetes Information Clearinghouse (NDIC)

Overweight

Defined by National Diabetes Information Clearinghouse (NDIC)

Fat

Defined by National Diabetes Information Clearinghouse (NDIC)

Calorie

Defined by National Diabetes Information Clearinghouse (NDIC)

Metformin

Defined by National Diabetes Information Clearinghouse (NDIC)

\section{Source}

National Diabetes Information Clearinghouse (U.S.). (2009). The diabetes dictionary. [Bethesda, Md.]: U.S. Dept. of Health and Human Services, National Institutes of Health, National Institute of Diabetes and Digestive and Kidney Diseases, National Diabetes Information Clearinghouse.

A study by the National Institute of Diabetes and Digestive and Kidney Diseases conducted from 1998 to 2001 in people at high risk for type 2 diabetes. All study participants had impaired glucose tolerance, also called pre-diabetes, and were overweight. The study showed that people who lost 5 to 7 percent of their body weight through a low-fat, low-calorie diet and moderate physical activity—usually walking for 30 minutes 5 days a week-reduced their risk of getting type 2 diabetes by 58 percent. Participants who received treatment with the oral diabetes drug metformin reduced their risk of getting type 2 diabetes by 31 percent. 